\title{
A Scoping Review on Biological Factors Associated with Interpersonal Violence in Adults with Severe Mental Illness
}

\author{
Maria Concepcion Moreno-Calvete* (iD) \\ Biocruces Bizkaia Health Research Institute, Basque Health System Bilbao, Bizkaia Mental Health Network, \\ Biscay, Spain
}

\begin{abstract}
Introduction: Knowledge about the biological basis of violence in people with severe mental illness is needed to formulate strategies in the management of violence. The aim of this study was to identify and describe the biological factors associated with interpersonal violence in adults with severe mental illness.

Methods: This scoping review was written with reference to the Preferred Reporting Items for Systematic reviews and Meta-Analyses extension for Scoping Reviews (PRISMA-ScR) Checklist. The literature search was conducted in MEDLINE (via PubMed), PsycINFO, SciELO, ProQuest and Epistemonikos until October 2020. Primary and Secondary studies in English or Spanish were included without restrictions as to date of publication. The information charted during the process included: authors; publication year; country; type of research; study population; biological factors; outcomes and key findings.
\end{abstract}

Results: Twenty-eight articles were included after the selection process. The most widely-studied population was people with schizophrenia. The biological factors studied in the articles are categorized into six groups: Serotonin function, genes, hormones, neuroactive steroids, brain function and anatomy, and immune factors. Several studies showed a relationship between different biological factors and interpersonal violence in people with severe mental illness.

Conclusion: This scoping review describes current research, and provides evidence on a biological basis. Nevertheless, further studies are needed to carry out research into biological factors associated with interpersonal violence in people with severe mental illness.

\section{Keywords}

Mental disorders (MeSH), Aggression, Violence, Biological factors

\section{Introduction}

Aggressive behavior is a complex phenomenon, sustained throughout evolution in most species including humans [1].

Evidence shows a higher prevalence of serious crime among people with schizophrenia and increased rates of convictions for violence $[2,3]$. In addition, it has been found that people with severe mental illness had perpetrated higher rates of violence and there is an increased risk for homicide in individuals with psychosis compared with the general population $[4,5]$.

Despite the prevalence of victimisation being higher than violence in people with severe mental illness, society has a negative, dangerous perception of people with mental illness and violence. Moreover, a study found that $5.3 \%$ of the estimated total social cost of violence was related to people with severe mental illness in England and Wales in 2015-16 [6-8].

There are different factors related to psychiatric in-patient aggression, such as being younger, male, being involved in involuntary admissions, having a greater number of previous admissions, a diagnosis of schizophrenia, a history of violence, a history of self-destructive behavior and a history of substance abuse [9]. In addition, some modifiable risk factors are associated with violence in psychosis, such as hostile behavior, recent drug, substance or alcohol misuse, non-adherence to psychological therapies, poorer impulse control scores, and non-adherence with pharmacological treatment [10].

*Corresponding author: Maria Concepcion Moreno-Calvete, Biocruces Bizkaia Health Research Institute, Basque Health System Bilbao, Bizkaia Mental Health Network, C/ María Díaz de Haro, 58, Bilbao 48010, Basque Country, Spain, Tel: +3494-47051-95, Fax: +34944705241

Accepted: February 02, 2021

Published online: February 04, 2021

Citation: Moreno-Calvete MC (2021) A Scoping Review on Biological Factors Associated with Interpersonal Violence in Adults with Severe Mental Illness. J Psychiatry Treat Res 3(1):37-43 
With regard to biological basis, various studies suggest biological factors have an effect on people with severe mental illness and aggressive behavior. Several studies have reported an association between gene polymorphisms and violence in people with schizophrenia, such as the Catechol-O-methyltransferase (COMT) polymorphism, the Methylenetetrahydrofolate reductase (MTHFR) gene polymorphism or functional polymorphisms of the dopamine receptor D4 (DRD4) and monoamine oxidase A (MAO-A) genes. Li, et al. found a relationship between Th17-related cytokine levels and the severity of schizophrenia and aggressive behavior. In addition, some studies found differences in gray matter volume, cortical morphology, and hippocampal volume in people with schizophrenia and violence [11-19].

Notwithstanding the existence of several studies on this topic, no scoping review has been found related to the biological basis of people with severe mental illness and aggressive behavior.

\section{Rationale}

The complexity of violence and its relationship with people with severe mental illness, its economic impact, the existence of evidence related to the role of biological factors in aggressive behavior and the absence of a scoping review on this topic calls for a study to provide knowledge on the biological basis of violence in this population that will help health practitioners to formulate strategies for the management of violence.

\section{Objective}

The aim of this study was to identify and describe the biological factors associated with interpersonal violence in adults with severe mental illness.

\section{Methodology}

\section{Study design and protocol registration}

This study was written with reference to the Preferred Reporting Items for Systematic reviews and Meta-Analyses extension for Scoping Reviews (PRISMA-ScR) Checklist [20]. The protocol was registered with the Open Science Framework (https:// osf.io/pw3a9/). This study did not require ethical approval as no patients or other participants were involved in this study.

\section{Identifying the research question}

This scoping review was conducted to answer the following research question: What are the biological factors associated with interpersonal violence in people with severe mental illness described in the literature?

\section{Information sources and search}

The literature search was conducted in the following databases: MEDLINE (via PubMed), PsycINFO, SciELO, ProQuest and Epistemonikos until October 2020. The systematic literature searches were conducted using a designed search strategy, and the search strategy used on Pubmed is given in Supplementary File 1 as an example. The combinations of keywords and search terms were adapted in other databases with no restriction as to year of publication. Zotero was used to import all studies and manage the references from the search.

\section{Study selection and screening process}

Eligibility criteria: The studies had to consider biological factors in interpersonal violence in people with severe mental illness in order to be included and violence had to include physical or verbal aggression.

The outcomes/measures of interest were:

- Biological factor: Any biological determination or analysis.

- Interpersonal violence: Physical or verbal aggression.

The inclusion criteria for severe mental illness was adults with a diagnosis of severe mental illness or any condition included in severe mental illness [21]: Schizophrenic disorder, schizoaffective disorder, schizotypal disorder, persistent or induced delusional disorder, bipolar disorder, obsessive-compulsive disorder, major depressive episode with psychotic features, recurrent major depressive disorders, atypical psychosis or other non-organic psychoses (according to international classification systems: Diagnostic and Statistical Manual of Mental Disorders or International Classification of Diseases). No restrictions were placed on the setting.

This scoping review included quantitative studies (primary original research) and systematic reviews/meta-analyses of quantitative studies (secondary research). When quantitative studies were present in a systematic review/meta-analysis, the review was selected instead of the primary study. All studies had to be published in Spanish or English. No restrictions were applied as to date of publication. The following study design was excluded from the scoping review: Narrative reviews.

\section{Study screening, selection and data extraction process}

The titles and abstracts of studies retrieved using the search strategy were screened to identify studies that meet the inclusion criteria and full texts were assessed for final inclusion/exclusion. Additionally, the reference list of articles was reviewed to identify other relevant studies. All relevant data was included to answer the scoping review questions. The information charted during the process included: authors; publication year; country; type of research; study population; biological factor; outcomes and key findings. In accordance with scoping review methodology, the quality of individual studies was not assessed.

\section{Results}

A total of 1734 articles were identified in the search, and 28 articles were included after the selection process (Figure 1). The selected studies were primary and secondary research from different states and the most widely studied population was people with schizophrenia [22].

\section{Biological factors associated with interpersonal violence}

The summary of the main results of twenty-eight articles 


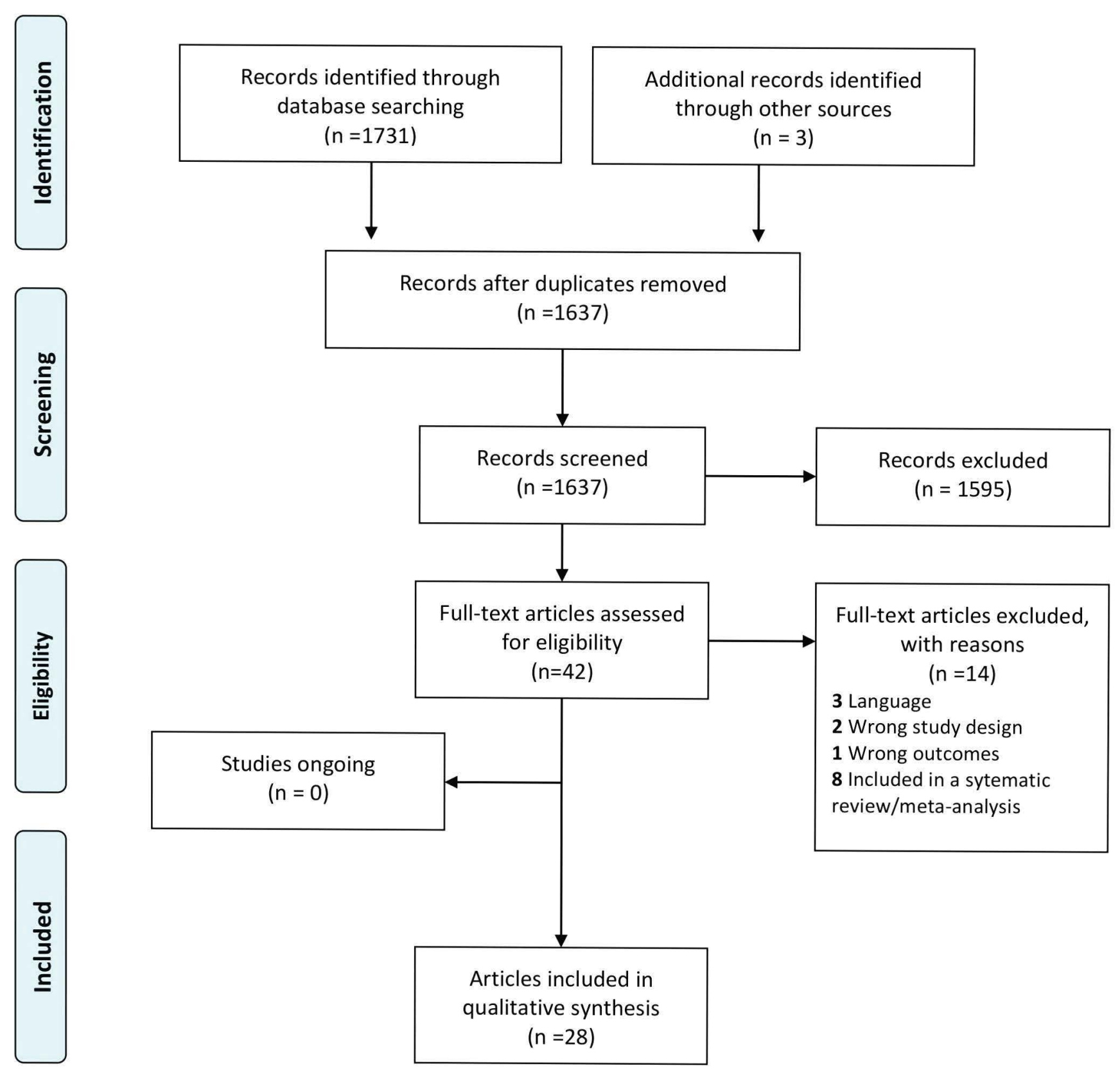

Figure 1: The Preferred Reporting Items for Systematic Reviews and Meta-Analyses (PRISMA) flow diagram for the scoping review, to illustrate the progress of studies through the selection process and screening [22].

are presented in a descriptive table (Table 1). The biological factors studied in the articles are categorized into six groups: (1) Serotonin function, (2) Genes, (3) Hormones, (4) Neuroactive steroids, (5) Brain function and anatomy, and (6) Immune factors.

\section{Serotonin function}

Maguire, et al. found no significant difference between aggressive and non-aggressive schizophrenic patients in the constant $\mathrm{K}_{\mathrm{d}}$ or $\mathrm{B}_{\max }$ for platelet $\left[{ }^{3} \mathrm{H}\right]$ paroxetine binding. Nevertheless, Modai, et al. found differences for $B_{\max }$ of $\left[{ }^{3} \mathrm{H}\right]$ paroxetine binding in the platelets of aggressive schizophrenic patients $[23,24]$.

Moreover, Barkan, et al. showed differences in the $\mathrm{V}_{\max }$ of $\left[{ }^{3} \mathrm{H}\right] 5-\mathrm{HT}$ to fresh lymphocytes and in the $\mathrm{K}_{\mathrm{m}}$ values of the $5-\mathrm{HT}$ to the transporter in currently aggressive patients with schizophrenia, and Chou, et al. found a significant association between aggressive behavior with serotonin transporter availability in the midbrain in bipolar disorder II patients $[25,26]$.

\section{Genes}

Catechol-O-methyl transferase (COMT) gene polymorphism: A meta-analysis (Bhakta, et al.) reported that the Met158 allele of the COMT gene was associated with higher risk for violence in schizophrenia. In addition, Singh, et al. showed that male patients with schizophrenia who carry the low activity Met allele of the Val158Met polymorphism of the 
COMT gene had an elevated risk of violence $[12,14]$.

Brain-derived neurotrophic factor (BDNF) Val66Met functional gene polymorphism: Chung, et al. and Guan, et al. found no significant difference in the genotype distribution or allelic frequency of the BDNF Val66Met polymorphism between the schizophrenic groups. Nonetheless, Spalletta, et al. observed that the BDNF Val66Met gene polymorphism is associated with a different level of aggressiveness in schizophrenic patients [27-29].

D-amino acid oxidase (DAO) and D-amino acid oxidase activator (DAOA) gene polymorphisms: Chung, et al. showed no significant differences in genotype distributions and allele frequencies of DAO and DAOA genes between groups [30].

Methylenetetrahydrofolate reductase (MTHFR) gene polymorphism: Dong, et al. suggested an association between the T-allele polymorphism of rs1801133 and the T-C haplotype of the MTHFR gene and susceptibility to aggressive behavior [13].

Dopamine receptor D4 (DRD4) and monoamine oxidase A (MAO-A) genes polymorphisms: Fresan, et al. found a significant association between patients with schizophrenia and aggressive behavior and the DRD4 gene, but no association between the MAO-A gene and aggressive behavior. However, there was a significant difference between the MAO-A/4R allele and verbal aggression [11].

Serotonin gene polymorphisms: Hong, et al. showed no significant difference in serotonin 1B Receptor (A-161T) gene polymorphism and Tsai, et al. found no significant difference in genotype or allele frequencies for serotonin 5-HT6 (C267T) polymorphism [31,32].

Tryptohan hydroxylase 1 (TPH1) A218C polymorphism: Kim, et al. found no significant association for TPH1 A218C polymorphism [33].

The T-cell receptor beta variable chain (TRBV): Li, et al. observed a significantly higher frequency of TRBV2 in the violent schizophrenia group [34].

\section{Hormones}

Das, et al. found a statistically significant correlation between cortisol variability and aggression [35].

\section{Neuroactive steroids}

Spalletta, et al. showed that increased aggressiveness was associated with increased $3 \alpha, 5 \alpha$ - tetrahydroprogesterone

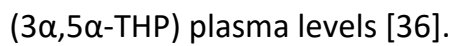

\section{Brain function and anatomy}

Two systematic reviews (Fjellvang, et al. and Widmayer, et al.) found volume differences in some areas such as prefrontal regions, temporal lobe, hippocampus, thalamus and cerebellum, lateral ventricles, amygdala, and putamen, and Tesli, et al. showed differences in hippocampal subfields and amygdala nuclei volumes in violent patients with schizophrenia $[16,19,37]$. In addition, Storverstre, et al. showed differences in cortical folding and cortical thickness in different cor- tex areas in violent schizophrenia patients and Schoretsanitis, et al. found differences of gray matter (GM) volume in the left inferior frontal gyrus $[17,18]$. Moreover, Garciá-Martí, et al. showed significant focal hyperactivations in patients with a greater association with violent behavior and Wong, et al. found a reduced [18F] fluorodeoxyglucose (FDG) uptake in the left anterior inferior temporal (LAIT) in violent patients $[38,39]$.

\section{Inmune factors}

$\mathrm{Li}$, et al. found that the Th17-related cytokine levels were related to the severity of schizophrenia and aggressive behavior and Zhang, et al. showed positive correlations between C-reactive protein (hSCRP) and interleukin (IL)-10 and aggression in patients with schizophrenia $[15,40]$. In addition, Das, et al. suggested that cytokines IFN-G and IL10 are both significantly associated with aggression and psychosis [41].

\section{Discussion}

The Catechol-O-methyl transferase (COMT) gene polymorphism and differences in brain function and anatomy were the most widely studied factors. These biological factors were evaluated by secondary research (systematic reviews and meta-analyses), which are at the top of the evidence pyramid, and the evidence shows a relationship between these factors and interpersonal violence.

Few studies found an association between the dopamine receptor D4 (DRD4) and monoamine oxidase A (MAO-A) gene polymorphisms, methylenetetrahydrofolate reductase (MTH$\mathrm{FR}$ ) gene polymorphism, T-cell receptor beta variable chain (TRBV), cortisol, $3 \alpha, 5 \alpha$ - tetrahydroprogesterone ( $3 \alpha, 5 \alpha-\mathrm{THP})$, Th17-related cytokines, C-reactive protein (hSCRP), interleukin (IL)-10 and aggressive behavior. On the other hand, the results on the brain-derived neurotrophic factor (BDNF) Val66Met functional gene polymorphism differ between studies and there is no evidence regarding D-amino acid oxidase (DAO) and D-amino acid oxidase activator (DAOA) gene polymorphisms, serotonin gene polymorphisms, tryptohan hydroxylase 1 (TPH1) A218C polymorphism and violent behavior. Not many studies have been found on these factors and all of them were primary studies, so it is still necessary to continue research in this area.

Despite the fact that the results derived from this scoping review are limited due to absence of evidence about some factors, the evidence shows the existence of an association between some biological factors and violent behavior in people with severe mental illness.

Regarding the limitations, as a scoping review, this study contributes to identifying biological factors in interpersonal violence in people with severe mental illness but does not assess the quality of the evidence. In addition, this study considered only studies published in English and Spanish, and it was carried out by one author. Notwithstanding, important data sources were searched and references of included studies were analysed to be included in this scoping review. It is important to consider the presence of primary study overlap in the included reviews and meta-analysis as a potential bias. 
Nonetheless, this scoping review describes current research and provides information that could help researchers in future studies on this topic.

A better understanding of the biological factors could help in developing strategies for managing violence, so further studies are needed to investigate biological factors associated with interpersonal violence in people with severe mental illness.

\section{Funding Statement}

This work was supported by the Department of Health of the Basque Government, Spain (Grant number 2017111101). The funder had no involvement in any aspect of the study or the decision to submit for publication.

\section{Competing Interests}

None declared.

\section{References}

1. Waltes R, Chiocchetti AG, Freitag CM (2016) The neurobiological basis of human aggression: $A$ review on genetic and epigenetic mechanisms. Am J Med Genet B Neuropsychiatr Genet 171: 650675.

2. Kim AM (2019) Crimes by people with schizophrenia in Korea: Comparison with the general population. BMC Psychiatry 19: 377.

3. Fazel S, Wolf A, Palm C, et al. (2014) Violent crime, suicide, and premature mortality in patients with schizophrenia and related disorders: A 38-year total population study in Sweden. Lancet Psychiatry 1: 44-54.

4. Choe JY, Teplin LA, Abram KM (2008) Perpetration of violence, violent victimization, and severe mental illness: Balancing public health concerns. Psychiatr Serv 59: 153-164.

5. Fazel S, Gulati G, Linsell L, et al. (2009) Schizophrenia and violence: systematic review and meta-analysis. PLoS Med 6: e1000120.

6. Tsigebrhan R, Shibre T, Medhin G, et al. (2014) Violence and violent victimization in people with severe mental illness in a rural low-income country setting: A comparative cross-sectional community study. Schizophr Res 152: 275-282.

7. Varshney M, Mahapatra A, Krishnan V, et al. (2016) Violence and mental illness: what is the true story? J Epidemiol Community Health 70: 223-225.

8. Senior M, Fazel S, Tsiachristas A (2020) The economic impact of violence perpetration in severe mental illness: A retrospective, prevalence-based analysis in England and Wales. Lancet Public Health 5: e99-106.

9. Dack C, Ross J, Papadopoulos C, et al. (2013) A review and meta-analysis of the patient factors associated with psychiatric in-patient aggression. Acta Psychiatr Scand 127: 255-268.

10. Witt K, van Dorn R, Fazel S (2013) Risk factors for violence in psychosis: Systematic review and meta-regression analysis of 110 studies. PloS One 8: e55942.

11. Fresan A, Camarena B, Apiquian R, et al. (2007) Association study of MAO-A and DRD4 genes in schizophrenic patients with aggressive behavior. Neuropsychobiology 55: 171-175.

12. Bhakta SG, Zhang JP, Malhotra AK (2012) The COMT Met158 allele and violence in schizophrenia: A meta-analysis. Schizophr Res 140: 192-197.

13. Dong ZQ, Tian YY, Guan X, et al. (2012) Genetic polymorphism of methylenetetrahydrofolate reductase and risk of aggressive behavior in schizophrenia. Psychiatry Res 200: 1082-1082.

14. Singh JP, Volavka J, Czobor P, et al. (2012) A meta-analysis of the Val158Met COMT polymorphism and violent behavior in schizophrenia. PLoS ONE 7: e43423.

15. Li H, Zhang Q, Li N, et al. (2016) Plasma levels of Th17-related cytokines and complement $\mathrm{C} 3$ correlated with aggressive behavior in patients with schizophrenia. Psychiatry Res 246: 700-706.

16. Widmayer S, Sowislo JF, Jungfer HA, et al. (2018) Structural magnetic resonance imaging correlates of aggression in psychosis: A systematic review and effect size analysis. Front Psychiatry 9: 217.

17. Schoretsanitis G, Stegmayer K, Razavi N, et al. (2019) Inferior frontal gyrus gray matter volume is associated with aggressive behavior in schizophrenia spectrum disorders. Psychiatry Res Neuroimaging 290: 14-21.

18. Storvestre GB, Valnes LM, Jensen A, et al. (2019) A preliminary study of cortical morphology in schizophrenia patients with a history of violence. Psychiatry Res Neuroimaging 288: 29-36.

19. Tesli N, van der Meer D, Rokicki J, et al. (2020) Hippocampal subfield and amygdala nuclei volumes in schizophrenia patients with a history of violence. Eur Arch Psychiatry Clin Neurosci 270: 771-782.

20. Tricco AC, Lillie E, Zarin W, et al. (2018) PRISMA extension for scoping reviews (PRISMA-SCR): Checklist and explanation. Ann Intern Med 169: 467-473.

21. (2009) Guideline development group of the Clinical Practice Guideline on Psychosocial Interventions in Severe Mental IIIness. Clinical Practice Guideline on Psychosocial Interventions in Severe Mental Illness. Quality Plan for the National Health System, Ministry of Health and Social Policy. Aragon Health Sciences Institute - I+CS. Clinical Practice Guidelines in the Spanish NHS: I+CS No $2007 / 05$.

22. Moher D, Liberati A, Tetzlaff J, et al. (2009) Preferred reporting items for systematic reviews and meta-analyses: The PRISMA statement. PLoS Med 6: e1000097.

23. Maguire K, Cheung P, Crowley K, et al. (1997) Aggressive behaviour and platelet 3-H-paroxetine binding in schizophrenia. Schizophr Res 23: 61-67.

24. Modai I, Gibel A, Rauchverger B, et al. (2000) Paroxetine binding in aggressive schizophrenic patients. Eur Psychiatry 17: 183.

25. Barkan T, Peled A, Modai I, et al. (2006) Serotonin transporter characteristics in lymphocytes and platelets of male aggressive schizophrenia patients compared to non-aggressive schizophrenia patients. Eur Neuropsychopharmacol 16: 572-579.

26. Chou YH, Lin CL, Wang SJ, et al. (2013) Aggression in bipolar II disorder and its relation to the serotonin transporter. J Affect Disord 147: 59-63.

27. Chung S, Chung HY, Jung J, et al. (2010) Association among aggressiveness, neurocognitive function, and the Val66Met polymorphism of brain-derived neurotrophic factor gene in male schizophrenic patients. Compr Psychiatry 51: 367-372.

28. Guan X, Dong ZQ, Tian YY, et al. (2014) Lack of association between brain-derived neurotrophic factor Val66Met polymor- 
phism and aggressive behavior in schizophrenia. Psychiatry Res 215: 244-245.

29. Spalletta G, Morris DW, Angelucci F, et al. (2010) BDNF Val66Met polymorphism is associated with aggressive behavior in schizophrenia. Eur Psychiatry 25: 311-313.

30. Chung S, Jung J, Chung HY, et al. (2007) No association between polymorphisms of DAO and DAOA genes and homicidal behaviors in Korean schizophrenia. Psychiatr Genet 17: 313.

31. Hong CJ, Pan GM, Tsai SJ (2004) Association study of onset age, attempted suicide, aggressive behavior, and schizophrenia with a serotonin $1 \mathrm{~b}$ receptor (A-161T) genetic polymorphism. Neuropsychobiology 49: 1-4.

32. Tsai SJ, Chiu HJ, Wang YC, et al. (1999) Association study of serotonin-6 receptor variant (C267T) with schizophrenia and aggressive behavior. Neurosci Lett 271: 135-137.

33. Kim YR, Lee JY, Min SK (2010) No evidence of an association between $\mathrm{A} 218 \mathrm{C}$ polymorphism of the tryptophan hydroxylase 1 gene and aggression in schizophrenia in a Korean population. Yonsei Med J 51: 27-32.

34. Li Q, Zhou J, Cao X, et al. (2018) Clonal characteristics of T-cell receptor repertoires in violent and non-violent patients with schizophrenia. Front Psychiatry 9: 403.
35. Das S, Sengupta S, Pathak K, et al. (2018) Aggression as an independent entity even in psychosis - The role of cortisol. Psychiatry Res 259: 405-411.

36. Spalletta G, Romeo E, Bonaviri G, et al. (2005) Preliminary evidence for an association between aggressive and hostile behavior and 3alpha,5alpha-tetrahydroprogesterone plasma levels in schizophrenia. J Psychiatry Neurosci JPN 30: 49-52.

37. Fjellvang M, Grøning L, Haukvik UK (2018) Imaging violence in schizophrenia: A systematic review and critical discussion of the MRI literature. Front Psychiatry 9: 333.

38. Garciá-Martí G, Martí-Bonmatí L, Aguilar EJ, et al. (2013) Functional neuroimaging in the study of aggressive behaviour in patients with schizophrenia. Rev Neurol 56: 193-199.

39. Wong MTH, Fenwick PBC, Lumsden J, et al. (1997) Positron emission tomography in male violent offenders with schizophrenia. Psychiatry Res Neuroimaging 68: 111-123.

40. Zhang $Q$, Hong W, Li H, et al. (2017) Increased ratio of high sensitivity C-reactive protein to interleukin-10 as a potential peripheral biomarker of schizophrenia and aggression. Int J Psychophysiol 114: 9-15.

41. Das S, Deuri SK, Sarmah A, et al. (2016) Aggression as an independent entity even in psychosis- the role of inflammatory cytokines. J Neuroimmunol 292: 45-51. 
Citation: Moreno-Calvete MC (2021) A Scoping Review on Biological Factors Associated with Interpersonal Violence in Adults with Severe Mental Illness. J Psychiatry Treat Res 3(1):37-43

\section{Additional file 1:}

\section{The search strategy used on Pubmed:}

((((")mental disorders"[MeSH Terms] OR ("mental"[All Fields] AND "disorders"[All Fields]) OR "mental disorders"[All Fields] OR ("severe"[All Fields] AND "mental"[All Fields] AND "disorder"[All Fields]) OR "severe mental disorder"[All Fields] OR (("sever"[All Fields] OR "severe"[All Fields] OR "severed"[All Fields] OR "severely"[All Fields] OR "severer"[All Fields] OR "severes"[All Fields] OR "severing"[All Fields] OR "severities"[All Fields] OR "severity"[All Fields] OR "severs"[All Fields]) AND ("mental disorders"[MeSH Terms] OR ("mental"[All Fields] AND "disorders"[All Fields]) OR "mental disorders"[All Fields] OR ("mental"[All Fields] AND "illness"[All Fields]) OR "mental illness"[All Fields])) OR ("schizophrenia"[MeSH Terms] OR "schizophrenia"[All Fields] OR "schizophrenias"[All Fields] OR "schizophrenia s"[All Fields]) OR ("psychotic disorders"[MeSH Terms] OR ("psychotic"[All Fields] AND "disorders"[All Fields]) OR "psychotic disorders"[All Fields] OR "psychosis"[All Fields]) OR ("bipolar disorder"[MeSH Terms] OR ("bipolar"[All Fields] AND "disorder"[All Fields]) OR "bipolar disorder"[All Fields] OR ("bipolar"[All Fields] AND "disorders"[All Fields]) OR "bipolar disorders"[All Fields]) OR ("schizoaffective"[All Fields] OR "schizoaffectives"[All Fields]) OR ("obsessive compulsive disorder"[MeSH Terms] OR ("obsessive compulsive"[All Fields] AND "disorder"[All Fields]) OR "obsessive compulsive disorder"[All Fields] OR ("obsessive"[All Fields] AND "compulsive"[All Fields] AND "disorder"[All Fields]) OR "obsessive compulsive disorder"[All Fields]) OR ("schizotypal personality disorder"[MeSH Terms] OR ("schizotypal"[All Fields] AND "personality"[All Fields] AND "disorder"[All Fields]) OR "schizotypal personality disorder"[All Fields] OR ("schizotypal"[All Fields] AND "disorder"[All Fields]) OR "schizotypal disorder"[All Fields]) OR ("schizophrenia, paranoid"[MeSH Terms] OR ("schizophrenia"[All Fields] AND "paranoid"[All Fields]) OR "paranoid schizophrenia"[All Fields] OR ("delusional"[All Fields] AND "disorder"[All Fields]) OR "delusional disorder"[All Fields])) AND ((("aggress"[All Fields] OR "aggressed"[All Fields] OR "aggressing"[All Fields] OR "aggression"[MeSH Terms] OR "aggression"[All Fields] OR "aggressions"[All Fields] OR "aggressive"[All Fields] OR "aggressiveness"[All Fields] OR "aggressively"[All Fields] OR "aggressives"[All Fields] OR "aggressivity"[All Fields] OR ("violence"[MeSH Terms] OR "violence"[All Fields] OR "violence s"[All Fields] OR "violences"[All Fields])) NOT ("drug"[All Fields] AND ("induce"[All Fields] OR "induced"[All Fields] OR "inducer"[All Fields] OR "inducers"[All Fields] OR "induces"[All Fields] OR "inducibilities"[All Fields] OR "inducibility"[All Fields] OR "inducible"[All Fields] OR "inducing"[All Fields]))) NOT (("alcohol s"[All Fields] OR "alcoholate"[All Fields] OR "alcoholates"[All Fields] OR "alcoholic s"[All Fields] OR "alcoholics"[MeSH Terms] OR "alcoholics"[All Fields] OR "alcoholic"[All Fields] OR "alcoholism"[MeSH Terms] OR "alcoholism"[All Fields] OR "alcoholisms"[All Fields] OR "alcoholism s"[All Fields] OR "alcoholization"[All Fields] OR "alcohols"[MeSH Terms] OR "alcohols"[All Fields] OR "ethanol"[MeSH Terms] OR "ethanol"[All Fields] OR "alcohol"[All Fields]) AND ("induce"[All Fields] OR "induced"[All Fields] OR "inducer"[All Fields] OR "inducers"[All Fields] OR "induces"[All Fields] OR "inducibilities"[All Fields] OR "inducibility"[All Fields] OR "inducible"[All Fields] OR "inducing"[All Fields]))) AND ("neurobiologies"[All Fields] OR "neurobiology"[MeSH Terms] OR "neurobiology"[All Fields] OR ("nervous system"[MeSH Terms] OR ("nervous"[All Fields] AND "system"[All Fields]) OR "nervous system"[All Fields]) OR ("epigenetical"[All Fields] OR "epigenetically"[All Fields] OR "epigenomics"[MeSH Terms] OR "epigenomics"[All Fields] OR "epigenetic"[All Fields] OR "epigenetics"[All Fields]) OR ("gene s"[All Fields] OR "genes"[MeSH Terms] OR "genes"[All Fields]) OR ("parietal lobe"[MeSH Terms] OR ("parietal"[All Fields] AND "lobe"[All Fields]) OR "parietal lobe"[All Fields]) OR ("cerebral cortex"[MeSH Terms] OR ("cerebral"[All Fields] AND "cortex"[All Fields]) OR "cerebral cortex"[All Fields]) OR ("hippocampus"[MeSH Terms] OR "hippocampus"[All Fields]) OR ("amygdala"[MeSH Terms] OR "amygdala"[All Fields] OR "amygdalas"[All Fields] OR "amygdala s"[All Fields] OR "amygdalae"[All Fields]) OR (("neural"[All Fields] OR "neuralization"[All Fields] OR "neuralize"[All Fields] OR "neuralized"[All Fields] OR "neuralizes"[All Fields] OR "neuralizing"[All Fields] OR "neurally"[All Fields]) AND ("circuitries"[All Fields] OR "circuitry"[All Fields])) OR ("temporal lobe"[MeSH Terms] OR ("temporal"[All Fields] AND "lobe"[All Fields]) OR "temporal lobe"[All Fields]) OR ("gyrus cinguli"[MeSH Terms] OR ("gyrus"[All Fields] AND "cinguli"[All Fields]) OR "gyrus cinguli"[All Fields]) OR ("prefrontal cortex"[MeSH Terms] OR ("prefrontal"[All Fields] AND "cortex"[All Fields]) OR "prefrontal cortex"[All Fields]) OR ("protein s"[All Fields] OR "proteinous"[All Fields] OR "proteins"[MeSH Terms] OR "proteins"[All Fields] OR "protein"[All Fields]) OR ("hormon"[All Fields] OR "hormonal"[All Fields] OR "hormonally"[All Fields] OR "hormonals"[All Fields] OR "hormone s"[All Fields] OR "hormones"[Pharmacological Action] OR "hormones"[MeSH Terms] OR "hormones"[All Fields] OR "hormone"[All Fields] OR "hormons"[All Fields]) OR ("infradian rhythm"[MeSH Terms] OR ("infradian"[All Fields] AND "rhythm"[All Fields]) OR "infradian rhythm"[All Fields]) OR ("circadian rhythm"[MeSH Terms] OR ("circadian"[All Fields] AND "rhythm"[All Fields]) OR "circadian rhythm"[All Fields]) OR (("brain"[MeSH Terms] OR "brain"[All Fields] OR "brains"[All Fields] OR "brain s"[All Fields]) AND ("dysfunctional"[All Fields] OR "dysfunctionals"[All Fields] OR "dysfunctioning"[All Fields] OR "dysfunctions"[All Fields] OR "physiopathology"[MeSH Subheading] OR "physiopathology"[All Fields] OR "dysfunction"[All Fields])))) NOT ("childhood"[All Fields] OR "childhoods"[All Fields])) NOT ("dementia"[MeSH Terms] OR "dementia"[All Fields] OR "dementias"[All Fields] OR "dementia s"[All Fields])) NOT ("personality disorders"[MeSH Terms] OR ("personality"[All Fields] AND "disorders"[All Fields]) OR "personality disorders"[All Fields] OR ("personality"[All Fields] AND "disorder"[All Fields]) OR "personality disorder"[All Fields])) NOT ("adolescences"[All Fields] OR "adolescency"[All Fields] OR "adolescent"[MeSH Terms] OR "adolescent"[All Fields] OR "adolescence"[All Fields] OR "adolescents"[All Fields] OR "adolescent s"[All Fields]) 